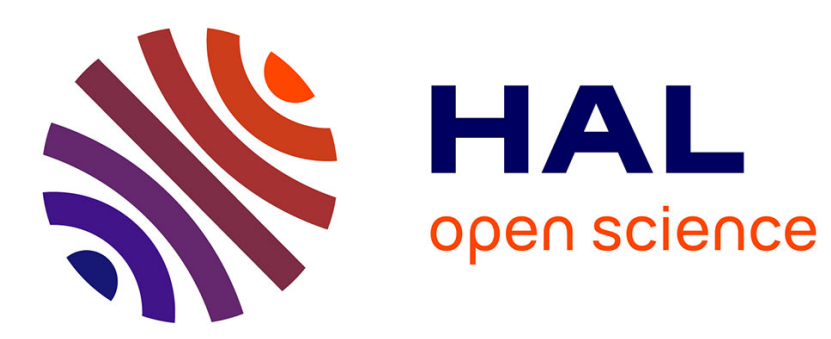

\title{
4He optical pumping with intensity modulated laser light
}

\author{
A. Cassimi, B. Cheron, J. Hamel
}

\section{To cite this version:}

A. Cassimi, B. Cheron, J. Hamel. 4He optical pumping with intensity modulated laser light. Journal de Physique II, 1991, 1 (2), pp.123-133. 10.1051/jp2:1991151 . jpa-00247502

\section{HAL Id: jpa-00247502 https://hal.science/jpa-00247502}

Submitted on 1 Jan 1991

HAL is a multi-disciplinary open access archive for the deposit and dissemination of scientific research documents, whether they are published or not. The documents may come from teaching and research institutions in France or abroad, or from public or private research centers.
L'archive ouverte pluridisciplinaire HAL, est destinée au dépôt et à la diffusion de documents scientifiques de niveau recherche, publiés ou non, émanant des établissements d'enseignement et de recherche français ou étrangers, des laboratoires publics ou privés. 
Classification

Physics Abstracts

$3280 \mathrm{~B}$

\title{
${ }^{4}$ He optical pumping with intensity modulated laser light
}

\author{
A Cassimı, B. Cheron (*) and J. Hamel \\ Laboratorre de Spectroscopıe Atomique (URA 19), ISMRa 14050 Caen Cedex, France \\ (Recelved 6 August 1990, accepted 12 November 1990)
}

\begin{abstract}
Résumé. - Les atomes métastables ${ }^{4} \mathrm{He}\left(2{ }^{3} \mathrm{~S}_{1}\right)$ sont pompés optıquement en lumière modulée en intensité L'utilisation, comme source de pompage, d'un laser monomode LNA pompé par une dıode laser permet l'étude de signaux de résonance prédits par la théorie du pompage optıque mais non encore observés Les résonances sont détectées sur le fassceau de pompage transmis à la fréquence de modulation et ses harmoniques L'application de cette méthode à la réalısation de magnétomètres de haute sensibilité est suggérée.
\end{abstract}

\begin{abstract}
He}\left(2{ }^{3} \mathrm{~S}_{1}\right)$ metastable atoms are optically pumped with intensity modulated light The use of a single mode diode-pumped LNA laser as the pumping source, allows the study of resonance signals predicted by the optical pumping theory but not yet observed Resonances are detected on the transmitted pumping beam at the modulation frequency and its harmonics Application of this method to high sensitivity magnetometers is suggested
\end{abstract}

\section{Introduction.}

In atomic optical pumping experıments, the magnetic resonance experiments have a particularly important place and lead to many applications such as high sensitivity magnetometers. In 1961, Bell and Bloom [1] suggested and published another very attractive method to induce driven spin precession and observe resonances without a radio-frequency magnetic field but only by intensity modulated pumping light at the Larmor frequency of atomic states in the static magnetic field This fine method gave only few experimental publications certainly because it was rather difficult to obtain a high modulation rate and to modulate the emitted intensity of the vapor lamp at high frequencies

In Bell and Bloom experiments [1,2], optical pumping of ground state Cs and $\mathbf{R b}$ atoms and metastable $2{ }^{3} \mathrm{~S}_{1} \mathrm{He}$ atoms is investigated. Resonance appears as a modification of the average intensity of the modulated pumping beam transmitted through the cell containing the atomic gas when the frequency of the modulation equals the Larmor frequency in a given static magnetic field Resonances at the modulation frequency and its harmonics were not experimentally investigated In Corney experiments ([3] and Refs. therein), Cd atoms are excited in the $5{ }^{3} \mathrm{P}_{1}$ state with an intensity modulated discharge lamp In the particular geometry used, resonance is observed as a modification of the modulation of the fluorescence

$\left.{ }^{*}\right)$ Also Unıversité de Caen, UFR de Sciences, 14032 Caen Cedex, France 
emitted at right angle when the frequency of the modulation is twice the Larmor frequency. Experiments are also reported where a R.F field and an intensity modulated pumping beam are applied simultaneously [4]

Recently we have developed a diode pumped LNA laser tunable on the $\left(2{ }^{3} \mathrm{~S}_{1}-2{ }^{3} \mathrm{P}\right){ }^{4} \mathrm{He}$ lines Using an optoelectronic modulator (acoustooptic), it is possible to modulate the laser beam with a modulation rate close to 1

In this paper we present our experiments where the resonances are optically detected. In particular, we detect the modulation induced in the pumping light beam after transmission through $\mathrm{He}$ atomic gas Another interesting method of detection has also been recently published [5], using optogalvanic effect These signals can be used in ${ }^{4} \mathrm{He}$ magnetometry. For high modulated pumping power, new resonances appear at multiple harmonics of the Larmor frequency. We systematically study the optical pumping intensity and polarization effects on the resonances and we present an experiment-theory comparison with a model using the density matrıx formalısm

\section{Experimental apparatus.}

The block diagram of the experimental apparatus is illustrated in figure 1 . The pumping light comes out from a LNA diode pumped laser [L] tuned on the $D_{0}\left(2{ }^{3} \mathrm{~S}_{1}-2{ }^{3} \mathrm{P}_{0}\right){ }^{4} \mathrm{He}$ transition at $10829 \mu \mathrm{m}$. This laser has been described in other papers [6,7] and gives about $10 \mathrm{~mW}$ in $\mathrm{CW}$ single mode emission The fine selection of the wavelength is provided by two intracavity solid étalons (thickness 200 and $500 \mu \mathrm{m}$ ) and a piezoelectric translator [Pz] which modifies the length of the laser cavity

The laser beam is intensity modulated by an acoustooptic modulator [AM] (Intra Action Corp. AOM-4 OR) driven by the signal processor [SP] (Intra Action model ME) The R F. excitation of the modulator at the frequency $N$ is given by a function generator [G] (PM 5192)

The modulated light is linearly or crrcularly polarized by the polarizer [P] and propagates through a spherical pyrex cell $[C](\Phi=4 \mathrm{~cm})$ containing ${ }^{4} \mathrm{He}$ atoms at a pressure of 15 Torr The beam diameter inside the cell is close to $5 \mathrm{~mm}$. The atoms are submitted to a weak H.F electrodeless electric discharge to create metastable $2{ }^{3} \mathrm{~S}_{1}$ excited states This cell is placed inside a cylindrical $\mu$-metal shield [S] (internal diameter $\cdot 50 \% \mathrm{~cm}$ and length $120 \mathrm{~cm}$ ) to avord fluctuations of the ambiant magnetic field A pair of Helmholtz coils [b] (diameter $40 \mathrm{~cm}$ ) provides the static magnetic field $\mathbf{B}$

A photodiode [PD1] detects the transmitted laser light through the helium cell and an electronic detection [D] allows measuring simultaneously the static value of the light intensity and the modulation of the light intensity at the frequency $N$ and at other harmonic components $2 N, 3 N$..

Another photodiode [PD2] detects the fluorescent light emitted by laser excited He atoms This photoelectric signal is used to lock the single mode laser frequency on the maximum of $D_{0}\left(2{ }^{3} \mathrm{~S}_{1}-2{ }^{3} \mathrm{P}_{0}\right)$ fluorescent light with the help of the piezoelectric translator [Pz]. In order to study in phase and in quadrature components of the modulated resonance signals we use a lock-in amplifier [A] (EGG Mod 5202) A frequency multıplier [M] generates harmonic frequencies $n N$ to monitor the lock-in amplifier reference. The amplitudes of in phase and in quadrature signals versus-magnetic field $B$ are recorded on a two channel chart recorder.

\section{Theory.}

The theory of optical pumping with modulated light has been established three decades ago $[2,8]$ We recall here the well known equations and discuss the approximations which apply to 


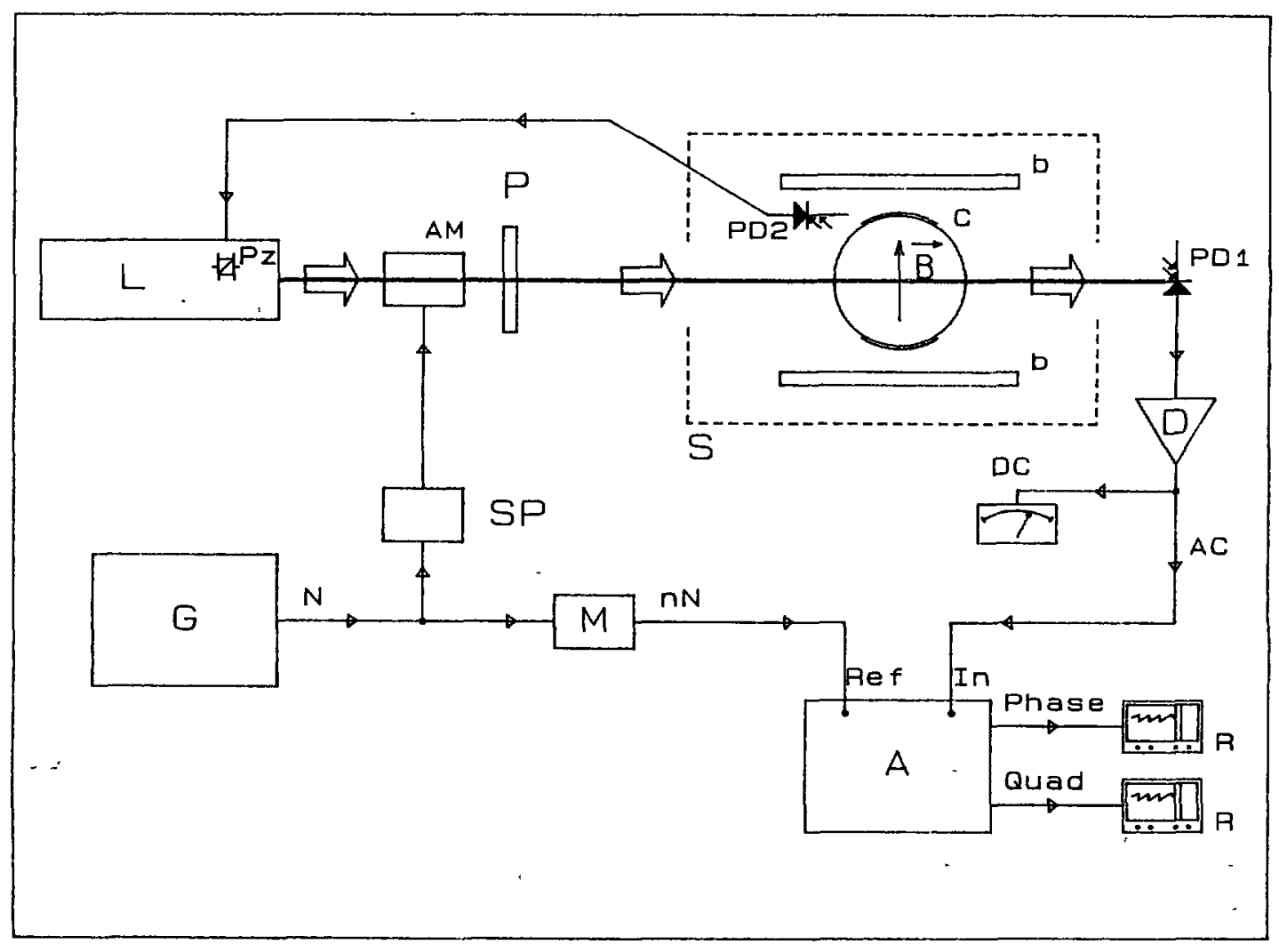

Fig 1 - Experımental set up L LNA tunable laser, AM : acoustooptıc modulator, $P$ polarızer, $\mathrm{C}$ helium cell ; b Helmholtz coils, S magnetic shield, PD1, PD2 photodetectors, D. amplifier; DC and AC are respectively the average value and the modulated part of the detected signal, $G$ function generator, S P signal processor driving the acoustooptic modulator AM, M. frequency multiplier; A lock-in amplifier, $\mathrm{R}$ chart recorder

the case of optical pumping of ${ }^{4} \mathrm{He}\left(2{ }^{3} \mathrm{~S}_{1}\right)$ with the $2{ }^{3} \mathrm{~S}_{1}-2{ }^{3} \mathrm{P}_{0}$ line The evolution of the standard irreducible components of the density matrices $\rho$ and $\rho_{\mathrm{e}}$ of a system of ${ }^{4} \mathrm{He}$ atoms in the $2{ }^{3} \mathrm{~S}_{1}$ metastable state and $2{ }^{3} \mathrm{P}_{0}$ state respectively is governed by:

$$
\begin{gathered}
\frac{\mathrm{d} \rho_{q}^{k}}{\mathrm{~d} t}=-J q \omega \rho_{q}^{k}-\Gamma \rho_{q}^{k}+\Lambda \delta_{k_{0}} \delta_{q_{0}}+\frac{1}{T_{\mathrm{p}}}\left(\Delta \rho_{q}^{k}\right)_{\mathrm{p}}+\frac{\Gamma_{\mathrm{e}} \rho_{\mathrm{e}}}{\sqrt{3}} \delta_{k_{0}} \delta_{q_{0}} \\
\frac{\mathrm{d} \rho_{\mathrm{e}}}{\mathrm{d} t}=-\Gamma_{\mathrm{e}} \rho_{\mathrm{e}}-\frac{\sqrt{3}}{T_{\mathrm{p}}}\left(\Delta \rho_{0}^{0}\right)_{\mathrm{p}}
\end{gathered}
$$

where $\omega$ is the Larmor angular frequency in the magnetic field $\mathbf{B}, \Gamma$ the relaxation constant of the metastable level (we assume a unique constant), $\Lambda$ is the creation rate of the metastable atoms by the discharge The last term in (1) represents the repopulation by spontaneous emission from the upper level $2{ }^{3} \mathrm{P}_{0}$ with $\Gamma_{\mathrm{e}}$, the radiative damping constant $\left(\Gamma_{\mathrm{e}} \cong 10^{7} \mathrm{~s}^{-1}\right)$. 
$\frac{1}{T_{\mathrm{p}}}\left(\Delta \rho_{q}^{k}\right)_{\mathrm{p}}$ is the pumping term where $T_{\mathrm{p}}$ is the pumping time. $\frac{1}{T_{\mathrm{p}}}$ is proportional to the power density of the pumping beam and can be expressed as :

$$
\frac{1}{T_{\mathrm{p}}}=\gamma(1+\varepsilon \cos \Omega t)
$$

where $\Omega$ is the intensity modulation angular frequency $(\Omega=2 \pi N)$ of the beam and $\varepsilon$ the modulation rate The power density is low enough to avoid absorption saturation However the average pumping constant $\gamma$ may be larger than the relaxation constant $\Gamma .\left(\Delta \rho_{q}^{k}\right)_{p}$ takes the general form [9] :

$$
\left(\Delta \rho_{q}^{k}\right)_{p}=\sum_{k^{\prime}, q^{\prime}} B_{q q^{\prime}}^{k k^{\prime}} \rho_{q^{\prime}}^{k^{\prime}}
$$

with :

$$
\begin{aligned}
& B_{q q^{\prime}}^{k k^{\prime}}=(-1)^{k+k^{\prime}-q+1} 3 \sqrt{2 k+1} \sqrt{2 k^{\prime}+1} \sum_{k^{\prime \prime}, q^{\prime \prime}} \sqrt{2 k^{\prime \prime}+1} \frac{1+(-1)^{k+k^{\prime}+k^{\prime \prime}}}{2} \times \\
& \times\left\{\begin{array}{rrr}
k & k^{\prime} & k^{\prime \prime} \\
1 & 1 & 1
\end{array}\right\}\left[\begin{array}{rrr}
k^{\prime \prime} & k^{\prime} & k \\
q^{\prime \prime} & q^{\prime} & -q
\end{array}\right] \Phi_{q^{\prime \prime}}^{k^{\prime \prime}}(e)
\end{aligned}
$$

where \{\} and [ ] are the $6 J$ and $3 j$ coefficients respectively and $\Phi_{q^{\prime \prime}}^{k^{\prime \prime}}(e)$ the irreducible component of the photon density matrix corresponding to the polarization vector $e$ of the pumping beam. The He vapor is supposed to be optically thin

The first simplification is to consider that the radiative damping constant $\Gamma_{\mathrm{e}}$ of the $2{ }^{3} \mathrm{P}_{0}$ state is much greater than the average pumping rate $\gamma$ so that $\rho_{\mathrm{e}}$ is negligible and $\rho_{0}^{0}$ can be considered as constant

We look for a general solution

$$
\rho_{q}^{k}(t)=\sum_{n=-\infty}^{+\infty} i^{(i n)} \alpha_{q}^{k} \mathrm{e}^{j n \Omega t}
$$

with ${ }^{(n)} \alpha_{0}^{0}=\frac{1}{\sqrt{3}} \delta_{n_{0}}$ (population normalized to 1) and the ${ }^{(n)} \alpha_{q}^{k}(k \neq 0)$ are solutions of the system :

$$
\begin{array}{r}
{[\Gamma+J(n \Omega+q \omega)]^{(n)} \alpha_{q}^{k}-\gamma \sum_{\substack{k^{\prime} \neq 0 \\
q^{\prime}}} B_{q q^{\prime}}^{k k^{\prime}}\left[{ }^{(n)} \alpha_{q^{\prime}}^{k^{\prime}}+\frac{\varepsilon}{2}\left(^{(n-1)} \alpha_{q^{\prime}}^{k^{\prime}}+{ }^{(n+1)} \alpha_{q^{\prime}}^{k^{\prime}}\right)\right]=} \\
\frac{\gamma}{\sqrt{3}} B_{q_{0}}^{k_{0}}\left[\delta_{n_{0}}+\frac{\varepsilon}{2}\left(\delta_{n_{1}}+\delta_{n=1}\right)\right]
\end{array}
$$

In the domain of low optical pumping power $(\gamma \ll \Gamma)$, the solution is found by taking only into account the equations with $n=0,1$ and neglecting all couplings with $|n|>1$.

For a given modulation angular frequency $\Omega$, resonance occurs if $n \Omega=q \omega$ ( $q$ integer). If $\Omega \gg \Gamma$, the different resonances are well separated and can be studied separately. The resonances are monitored by measuring the absorption of the pumping beam through the cell. This absorption is proportional to the' quantity $L_{\mathrm{A}}$ :

$$
L_{\mathrm{A}}=\frac{1}{T_{\mathrm{p}}} \sum_{k, q}(-1)^{k+q} \Phi_{-q}^{k} \rho_{q}^{k}
$$


For a linear polarization vector e making an angle $\theta$ with $\mathbf{B}$, resonances at $\omega=0$, $\Omega / 2$ and $\Omega$ take the following form (restricted to the lowest order in average optical power):

$$
\begin{aligned}
L_{\AA}^{(0)} & =-g^{2}(1+\varepsilon \cos \Omega t)\left[2 \sin ^{2} \theta \cos ^{2} \theta \frac{1}{1+u^{2}}+\frac{\sin ^{4} \theta}{2} \times \frac{1}{1+4 u^{2}}\right] \\
L_{\AA}^{(\Omega / 2)} & =-g^{2} \varepsilon(1+\varepsilon \cos \Omega t) \frac{\sin ^{4} \theta}{4}\left[\frac{\cos \Omega t+(X-2 u) \sin \Omega t}{1+(X-2 u)^{2}}\right] \\
L_{\AA}^{(\Omega)} & =-g^{2} \varepsilon(1+\varepsilon \cos \Omega t) \sin ^{2} \theta \cos ^{2} \theta\left[\frac{\cos \Omega t+(X-u) \sin \Omega t}{1+(X-u)^{2}}\right]
\end{aligned}
$$

Similarly, for a circularly polarized beam with a propagation direction making an angle $\theta$ with $\mathbf{B}$, we obtain

$$
\begin{aligned}
L_{\AA}^{(0)} & =-g^{2}(1+\varepsilon \cos \Omega t)\left[\frac{\sin ^{2} \theta\left(1+\cos ^{2} \theta\right)}{2} \frac{1}{1+u^{2}}+\frac{\sin ^{4} \theta}{8} \times \frac{1}{1+4 u^{2}}\right] \\
L_{\AA}^{(\Omega / 2)} & =-g^{2} \varepsilon(1+\varepsilon \cos \Omega t) \frac{\sin ^{4} \theta}{16}\left[\frac{\cos \Omega t+(X-2 u) \sin \Omega t}{1+(X-2 u)^{2}}\right] \\
L_{\AA}^{(\Omega)} & =-g^{2} \varepsilon(1+\varepsilon \cos \Omega t) \frac{\sin ^{2} \theta\left(1+\cos ^{2} \theta\right)}{4}\left[\frac{\cos \Omega t+(X-u) \sin \Omega t}{1+(X-u)^{2}}\right]
\end{aligned}
$$

where $u=\omega / \Gamma, X=\Omega / \Gamma, g=\gamma / \Gamma$

Resonances appear on the transmitted beam as modulation at null, $\Omega$ and $2 \Omega$ frequencies. When the pumping constant $\gamma$ is no longer negligible with respect to the relaxation constant $\Gamma$, radiative broadening occurs The resonances take a more complicated form and the corresponding expressions may be obtained by a development to a higher order in $g$ or numerically evaluated. Modulations of the transmitted beam at higher harmonics are found by including equations with $|n|>1$ For example, beam modulation at the third harmonic exhibits a resonance at $\omega=\Omega$ and, for a linear polarization vector, -the corresponding expression (restricted to the lowest power in $g$ ) is given by :

$$
L_{\mathrm{A}}=g^{3} \varepsilon^{3} \frac{3 \sin ^{4} \theta \cos ^{2} \theta}{16}\left[\frac{\left[1-2(X-u)^{2}\right] \cos 3 \Omega t+3(X-u) \sin 3 \Omega t}{\left[1+(X-u)^{2}\right]\left[1+4(X-u)^{2}\right]}\right]
$$

\section{Experimental results.}

41 RESONANCE POSITIONS. - Figure 2 shows the amplitude of the modulation of the transmitted beam at angular frequency $\Omega(N=250 \mathrm{kHz})$ and $2 \Omega$ versus magnetic field $B$ cosine component (a) and sine component (b) The beam is linearly polarized with the electric field e making an angle $(\mathbf{e}, \mathbf{B})=45^{\circ}$ and the modulation rate is $\varepsilon=068$ As expected from relations $(9,10,11)$, resonances are observed at magnetic field $B=B_{0}$ and $B_{0} / 2$ corresponding to Larmor frequencies $\omega=\Omega$ and $\Omega / 2$ and as intensity modulation at the two frequencies $\Omega$ and $2 \Omega$ At $\omega=0(B=0)$, a significant resonance is observed only on the cosine component of the modulation at $\Omega$ The small resonances visible at $B=0$ on the modulation at the frequency $2 \Omega$ may be due to a small intensity modulation at $2 \Omega$ of the incident beam caused by non-linearity in the acoustooptic modulator. Relative amplitudes of the resonances are in good agreement with the theory.

4.2 RESONANCES AT MULTIPLE HARMONiCS. - Modulations of the transmitted beam have been observed up to the tenth harmonic Some of them at $\omega=\Omega$ are represented in figure 3 


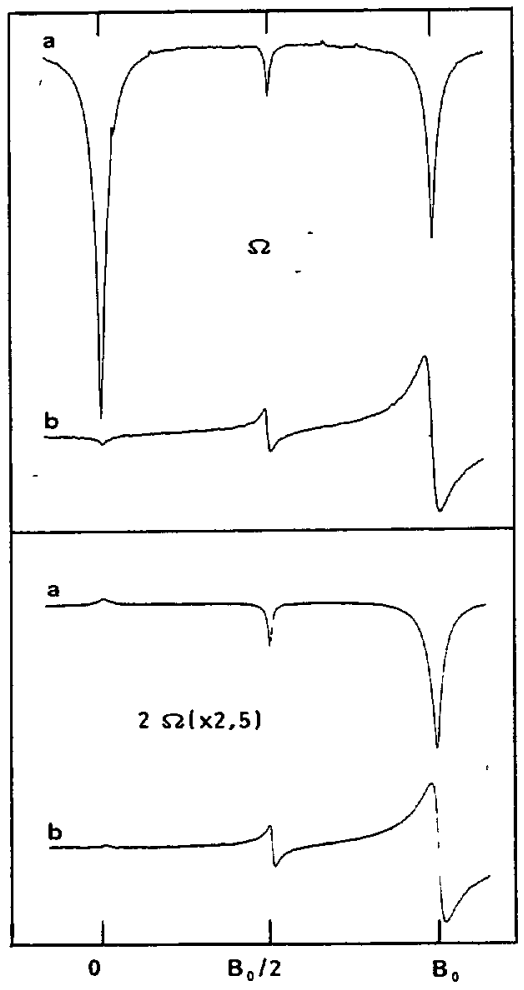

Fig 2 - Amplitude of the modulation of the transmitted beam at angular frequency $\Omega$ (top) and $2 \Omega$ (bottom) versus magnetic field $B$ with the cosine components on the left and the sine components on the right Resonances are observed at field intensities $B=0, B_{0} / 2, B_{0}$ corresponding to Larmor frequencies $\omega=0, \Omega / 2, \Omega$ respectively

with the amplitude of the cosine component on the left and of the sine component on the right. In the following, we have analysed the amplitude of the resonances versus modulation rate, the amplitude and width versus optical power and finally, the angular dependance of the signals

a) Resonance amplitude versus modulation rate $\varepsilon$ relation (11) shows that for $\omega=\Omega$, the amplitudes of the cosine components $S(\Omega)$ and $S(2 \Omega)$ at frequencies $\Omega$ and $2 \Omega$ are proportional to $\varepsilon$ and $\varepsilon^{2}$ respectively so that

$$
S(\Omega)=\frac{2 S(2 \Omega)}{\varepsilon} .
$$

The two quantities $S(\Omega)$ and $\frac{2 S(2 \Omega)}{\varepsilon}$ are plotted versus $\varepsilon$ in figure 4 in the case of a linearly polarized beam with $\theta=45^{\circ}$ and an average power $\bar{P}=0.55 \mathrm{~mW}$. As predicted, the experimental values fit well on a straight line The two lines may be considered as identical we note that relation (11) is valid only at low optical power As the optical power increases, we observe that the two quantities $S(\Omega)$ and $2 S(2 \Omega) / \varepsilon$ are still represented by straight lines but with different slopes. Similar conclusions are found for a circularly polarized beam

b) Amplitude and width versus optical power the widths of the resonances at $\omega=\Omega$, observed. on the first three harmonics are represented versus optical power $\bar{P}$ in figure 5. At zero optical power, the widths at the two frequencies $\Omega$ and $2 \Omega$ have a 


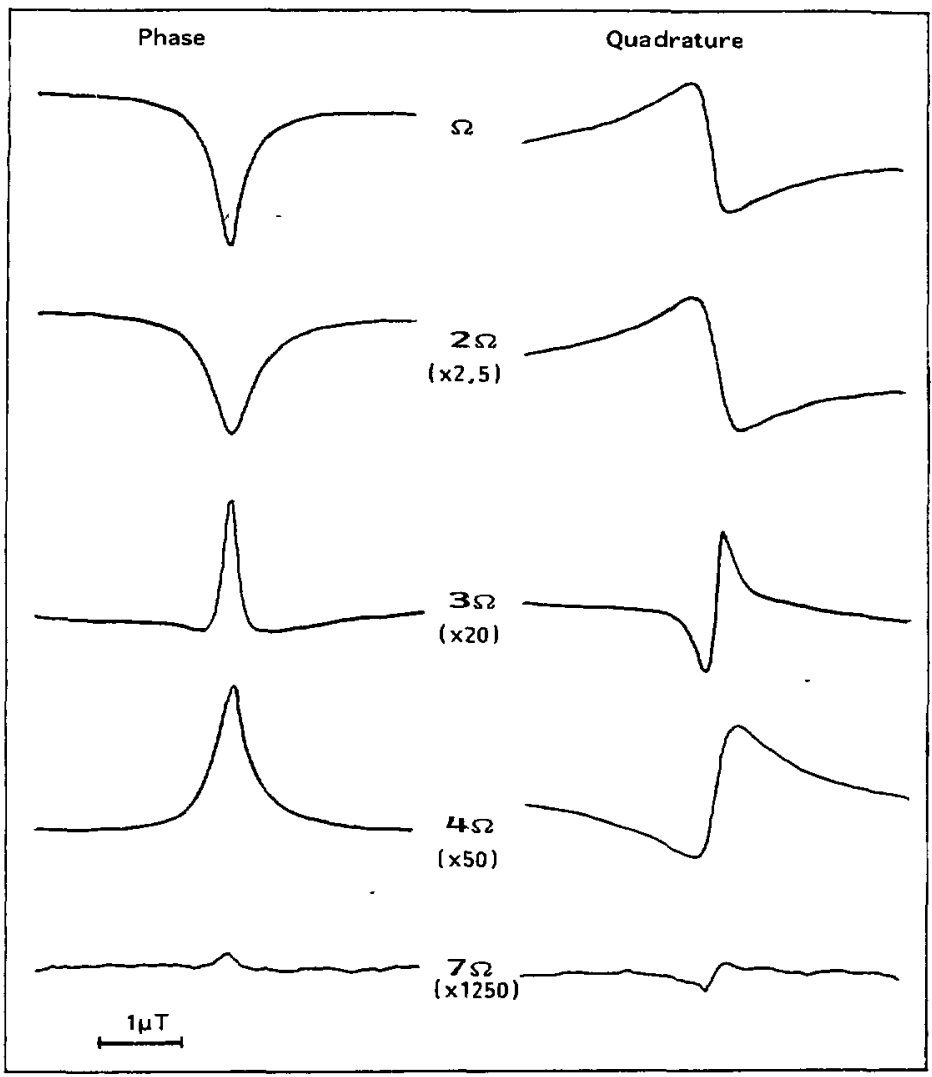

Fig 3 - Resonance amplitudes versus mangetıc field $B$ of several harmonics of the modulation angular frequency $\Omega(N=250 \mathrm{kHz})$ The resonances are observed at the Larmor frequency $\omega=\Omega$ The cosine components (in phase) are represented on the left and the sine components (in quadrature) are on the right The electric field $e$ of the linearly polarized laser beam is such that $(e, B)=45^{\circ}$ Modulation rate $\varepsilon=08$.

common value equal to $2 \Gamma(8 \mathrm{kHz}$ in frequency units) The width at the frequency $3 \Omega$ is much larger than expected from relation (15) Actually, the resonance curve is not a pure lorentzian curve (see Fig 3) and the calculated width of the cosine resonance at zero power is equal to $06 \Gamma$ The experimental value is somewhat higher and this discrepancy is due to a magnetic field inhomogeneity over the volume of the helium cell

The widths of the 3 resonances have not the same dependence versus $\bar{P}$ This is in fairly good agreement with the result of a numerical resolution of equation (7) represented by solid lines in figure 5 The parameters needed for the calculation are experimentally obtained ( $\Gamma=4 \mathrm{kHz}, \varepsilon=0.72$, linear polarization with $\left.\theta=45^{\circ}\right)$ and the ratio $\gamma / \bar{P}$ is obtained by fitting experimental and calculated widths for $\bar{P}=2 \mathrm{~mW}$ on the $\Omega$ curve The physical meaning of the difference between the slopes of the curves corresponding to the frequencies $\Omega$ and $2 \Omega$ is not evident this difference is explained by the calculation if we take into account terms with $|n|=2$ in the expansion of the density matrix elements (6)

The amplitudes of the 3 resonances are represented versus $\bar{P}$ in a log-log scale in figure 6 together with the result of a calculation (solid lines) using the same parameters as before. In the zero power limit, the amplitudes at the two frequencies $\Omega$ and $2 \Omega$ are predicted to behave 


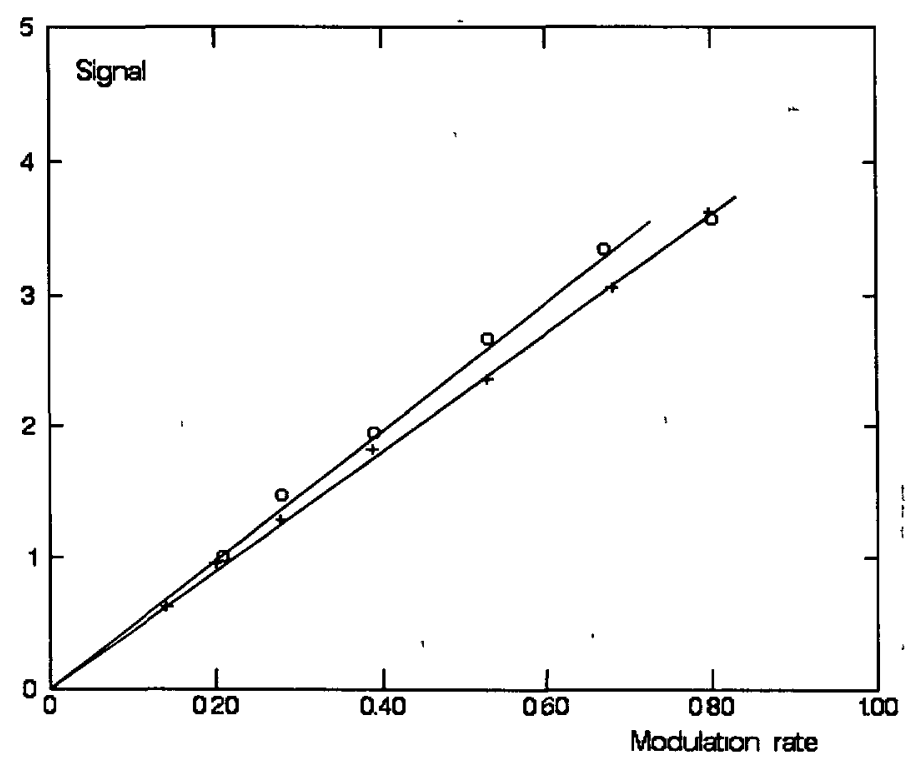

Fig $4-S(\Omega)(\square)$ and $\frac{2 S(2 \Omega)}{\varepsilon}(+)$ versus modulation rate $\varepsilon S(\Omega)$ and $S(2 \Omega)$ are the resonance amplitudes of the $\Omega$ and $2 \Omega$ harmonics observed at the Larmor angular frequency $\omega=\Omega$ and at constant average optical power $\bar{P}=055 \mathrm{~mW}$ The laser beam is linearly polarized.

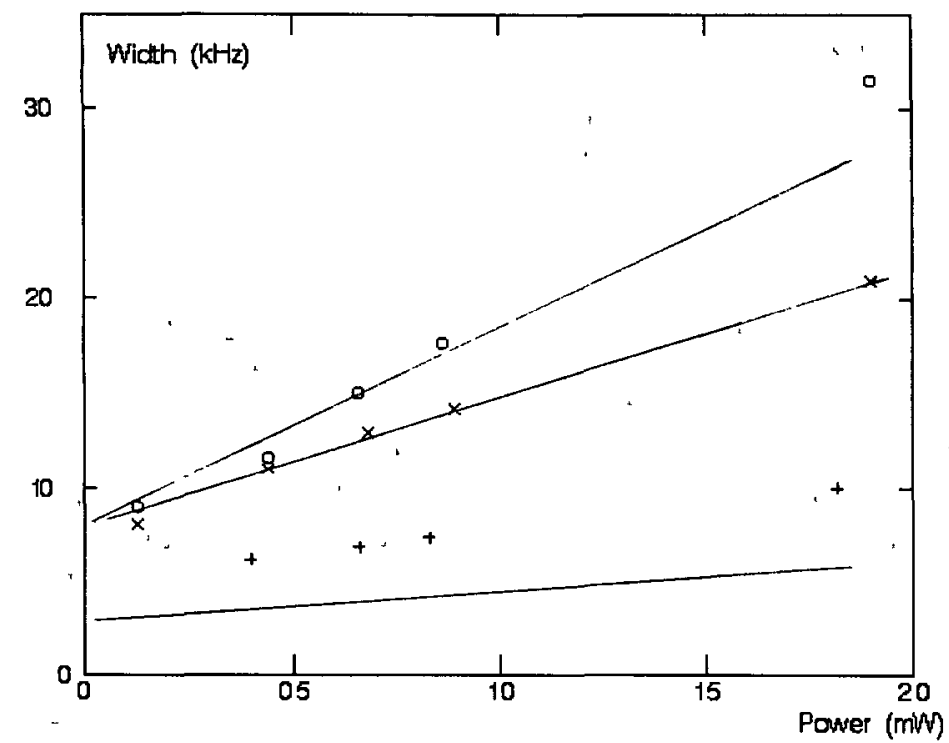

Fig. 5 - Widths (1n frequency units) of the resonances at Larmor frequency $\omega=\Omega$, observed on the first three harmonics of the modulation frequency $\Omega$, versus average optical power $\bar{P} \cdot \Omega(\times), 2 \Omega(\square)$, $3 \Omega(+)$ The laser beam is linearly polarized and the modulation rate is equal to 072 Full lines are calculated (see text for detals) 


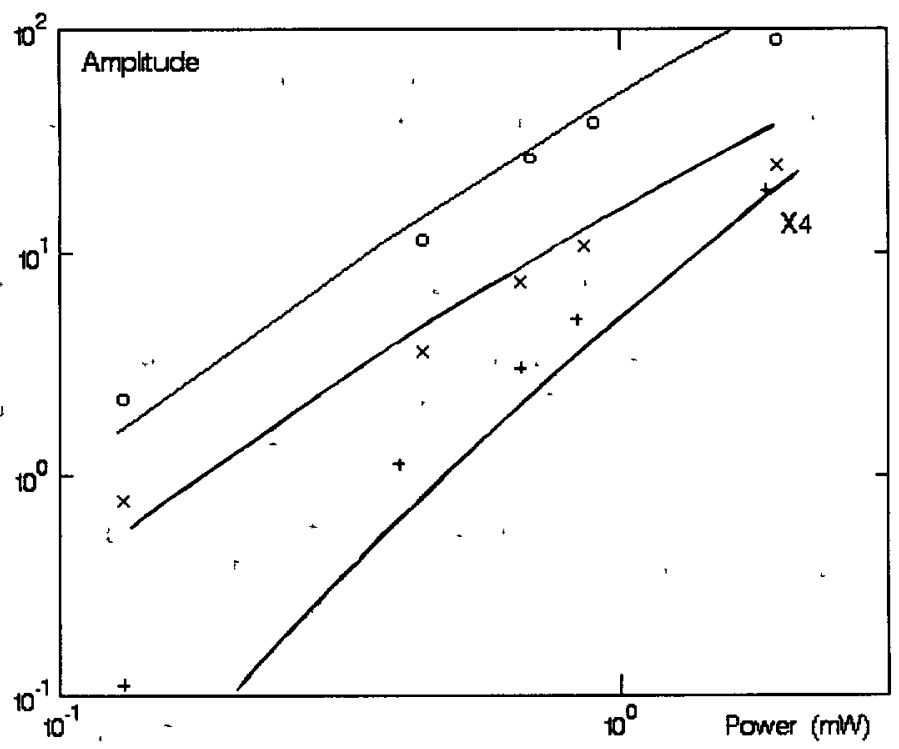

Fig 6 - Amplitudes of the resonances at Larmor frequency $\omega=\Omega$, observed on the first three harmonics of the modulation frequency $\Omega$ versus average optıcal power $\bar{P}: \Omega(\times), 2 \Omega(\square), 3 \Omega(+)$ (Linear polarization, modulation rate $\varepsilon=072$ ) Full lines are calculated

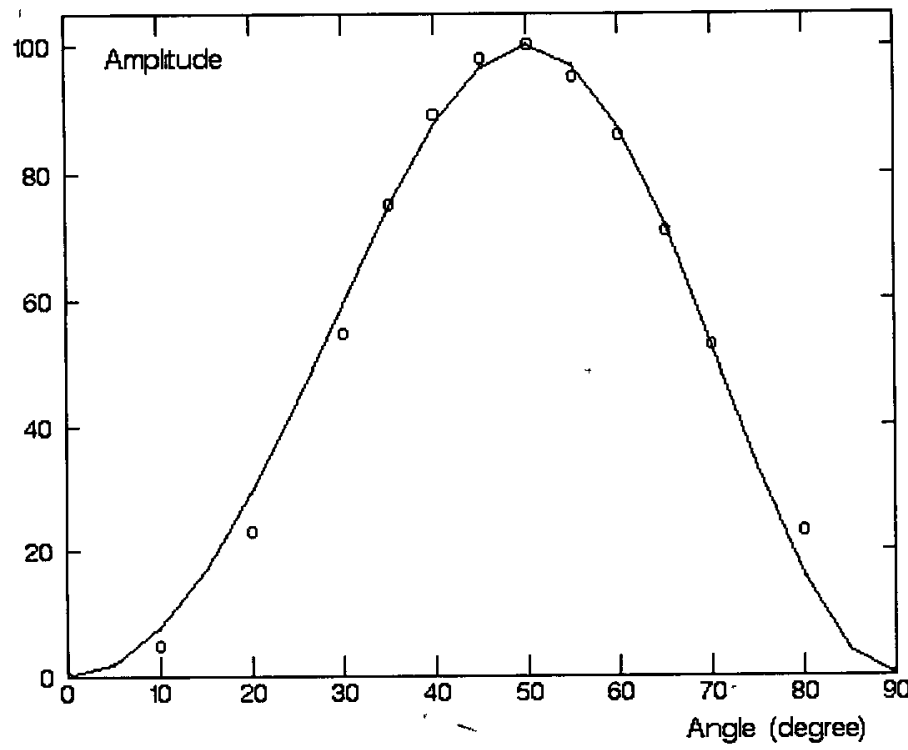

Fig 7 - Amplitude of the intensity modulation at frequency $\Omega$ observed at $\omega=\Omega$ versus angle $\theta$ between the electric field $\mathbf{e}$ of the linearly polarized beam and the magnetic field $\mathbf{B}$. The modulation rate is 07 Full line is calculated 
like $(\bar{P})^{2}$, while the amplitude at the frequency $3 \Omega$ behave like $(\bar{P})^{3}$. The experimental results agree qualitatively with the calculation. Width and amplitude are important characteristics in magnetometry application: the sensitivity of the magnetometer is proportional to the ratio amplitude over width.

c) Spatial anisotropy figure 7 represents the amplitude of the resonance at $\omega=\Omega$ versus angle $\theta$ between the magnetic field and the polarization vector of the beam. At low optical power, spatial anısotropy is described by the geometrical factor in relation (11). As expected, the experimental signal vanishes for $\theta=0$ and $\theta=\pi / 2$ and is maximum for $\theta$ close to $45^{\circ}$. The anisotropy profile is lightly modified when optical power increases The solid line is the result of a calculation with $\gamma / \Gamma=0.6$. This parameter is estimated from the measurement of the width of the resonance and with the help of figure 5

Similarly, figure 8 represents the amplitude of the resonance at $\omega=\Omega$ in the case of circular polarization $\theta$ is the angle between the magnetic field and the propagation direction of the beam The solid line is the result of a calculation with $\gamma / \Gamma=1$. In both cases, theory and experiment are in good agreement.

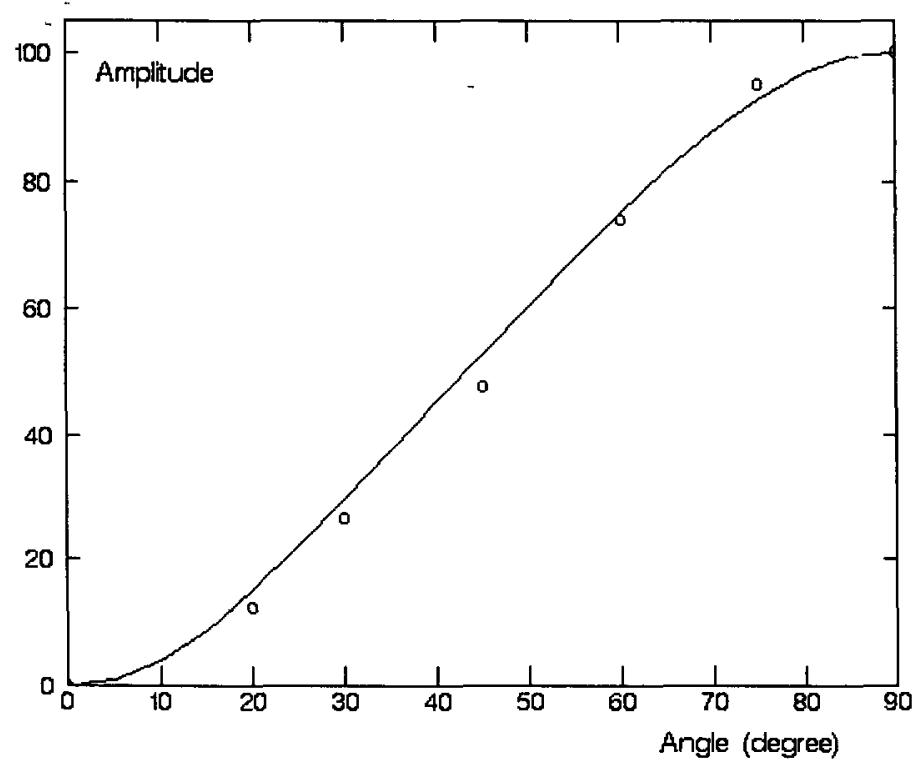

Fig 8 - Amplitude of the intensity modulation at frequency $\Omega$ observed at $\omega=\bar{\Omega}$ versus angle $\theta$ between the propagation direction of the circularly polarized beam and the magnetic field $B$ The modulation rate is 09 Full line is calculated

\section{Conclusion.}

The recent possibility to use a laser in helium optical pumping allows now the study of interesting theoretically predicted effects difficult to obtain with a lamp It has been shown that when optical pumping is achieved with only one light beam, the best efficiency is obtained when using one of the three $D$ lines this can be done only with a laser Two other advantages of the laser over the lamp are high intensity and high frequency intensity modulation capability with an electrooptic device 
Exploitation of these new resonance signals may be possible in magnetometry and a systematic comparison between the signal to noise ratio related to the magnetometer sensitivity, obtained with magnetic resonance on -one hand and with intensity modulated pumping method on the other hand, are in progress The obvious great advantage of the second method is the absence of R.F magnetic field. As a consequence, the resonances are free from R F. broadening

At the present time, we are studying another new optical pumping method in which the polarization of the beam is modulated Interesting large signals are also obtained.

\section{References}

[1] Bell W E and Bloom A. L, Phys Rev. Lett 6 (1961) 280

[2] Bloom A. L and Bell W E, Advances in Quantum Electronics (Columbia University Press, New York and London) 1961, p 224

[3] Corney A, $J$ Phys B 1 (1968) 458

[4] Novikov L. N and Pokazan'ev V G, Sov Phys JETP 26 (1968) 438

[5] Schearer L D and Tin P., $J$ Opt Soc Am B 6 (1989) 1771

[6] Aubert J J , Wyon Ch, Cassimi A., Hardy V, Hamel J , Opt Comm 69 (1989) 299

[7] Cassimi A, Thesis, Caen (1989) unpublished.

[8] Pokazan'ev V G, Opt Spectrosc 24 (1968) 182

[9] Faroux J P., Thesis, Paris (1969) unpublished (mıcrofilm n AO 3807, CNRS, 15 qual Anatole France, 75007 Paris). 